\title{
1 AND 5 DAY DIFFERENTIAL INSAR UNDER CROSSING ORBITS WITH TERRASAR-X
}

\author{
Steffen Wollstadt, Paco López-Dekker, Pau Prats-Iraola, Francesco De Zan, Thomas Busche \\ and Gerhard Krieger
}

\author{
German Aerospace Center (DLR), Microwaves and Radar Institute, Oberpfaffenhofen, Germany \\ Email: steffen.wollstadt@dlr.de
}

\begin{abstract}
This paper presents investigations on a one year time series of crossing orbit differential interferometry SAR acquisitions with a 1-, 5and 6-days temporal baseline. The conditions for crossing orbit interferometry are briefly discussed as the requirement of a common ground spectrum has to be satisfied. The uniquely short revisit times give the opportunity to perform interferometry on a glacier area, i.e. the Ronne ice-shelf, in X-band. The coherence results over one year as well as surface velocity measurements are shown and discussed.
\end{abstract}

Index Terms - Crossing orbits, SAR, interferometry, Ronne ice-shelf, TerraSAR-X.

\section{INTRODUCTION}

In spaceborne differential Synthetic Aperture Radar (SAR) interferometry short revisit times are of interest for all change detection applications sensitive to temporal decorrelation. Repeat-pass interferometry with current spaceborne SAR systems is typically dependent on the revisit time cycle. The regular repeat cycle of some former, current and up-coming spaceborne SAR missions are listed in Table 1. Currently, TerraSAR-X (TSX) and TanDEM-X (TDX) have the shortest repeat-pass time with 11 days considering only single satellites in case of missions with more than one satellite like CosmoSkymed.

Temporal decorrelation has a large impact on the results of differential interferometric monitoring of areas with temporally changing scatterers, like forests and ice. Usually, differential interferometry is used to detect changes due to surface deformation or velocities. Temporal changes of the scatterers result in a random interferometric phase and, hence, in errors in the final results. Longer revisit times and higher operating frequencies result typically in stronger coherence losses. Therefore, a decrease of the revisit time is especially important for X-band systems, which are more sensitive to surface changes.

It has been shown in [1] and [2] that 1- and 5-day quasi repeatpass interferometry is possible under crossing orbits with TSX/TDX. Although in a sun-synchronous orbit the acquisition area is restricted to high latitudes, i.e. polar regions, studying and monitoring of ice and glaciers w.r.t. short-term coherence is feasible. Note, that the crossing orbits introduce also additional aspects like varying volumetric coherence due the azimuth varying baseline.

In Section 2 a short revision of crossing orbit interferometry is given and the Ronne ice-shelf test site in Antarctica is presented. Section 3 shows and discusses the interferometric results of a crossing orbit acquisition triplet. In Section 4 the results of the interferometric time series are presented and different aspects of the coherence as well as surface velocity measurements are shown. Section 5 concludes the paper.
Table 1. Spaceborne SAR satellite repeat cycles

\begin{tabular}{lcc}
\hline SAR mission & Repeat cycle & Frequency \\
\hline TerraSAR-X/TanDEM-X & 11 days & X-band \\
Sentinel 1(A) & 12 days & C-band \\
Cosmo-Skymed & 16 days & X-band \\
Radarsat-2 & 24 days & C-band \\
Envisat/ASAR, ERS-1/2 & 35 days & C-band \\
ALOS/Palsar & 46 days & L-band \\
\hline
\end{tabular}

\section{CROSSING ORBIT INTERFEROMETRY}

\subsection{Acquisition Geometry and Satellite Configuration}

Fig. 1a shows the acquisition geometry for the interferograms with a 1-, 5- and 6-day time-lag. Note, that the 5- and 6-day orbit track geometries are ideally symmetric w.r.t. to interferometric information. Nevertheless differences can occur due to temporal changes. Due to the crossing orbits, special geometric requirements have to be considered in order to satisfy the spectral interferometric constraints, which are namely a common ground spectrum in azimuth and range. The natural inherited spectral shifts of the non-parallel orbits can be compensated by this acquisition geometry. The choice of the 1-, 5and 6-day lag results also from the spectral requirements, since this are the orbit tracks with the smallest crossing angles. Limitations for successful interferometry under crossing orbits are the critical baseline and the maximum azimuth antenna steering angle of the SAR instrument. Details of the geometry are described in [1] and details of the spectral theory can be found in [3] and [4]. The unusual acquisition geometry leads at the same time to strongly varying baselines (cf. Fig. 1b). This limits the possible observable area w.r.t. coherence (cf. Fig. 2) but simultaneously provides the opportunity for volume decorrelation studies or fine topography estimation (roughness). The idea of not using exactly repeat-pass acquisitions, i.e. parallel ground tracks, for interferogram generation was first reported in [5].

\subsection{The Ronne Ice-Shelf Testsite}

A reasonably flat test site located on the Ronne ice-shelf in Antarctica with center coordinates of $78^{\circ} \mathrm{S} / 56.5^{\circ} \mathrm{W}$ was chosen due to the high latitude in the southern hemisphere. Furthermore, the ice-shelf is known to be flowing and is therefore even more interesting w.r.t. short-term monitoring. A time series over one year was planned and around one acquisition triplet per month were set up. All acquisition dates are shown in Table 2 . They were executed with $150 \mathrm{MHz}$ pulse bandwidth in order to obtain a swath width of around $30 \mathrm{~km}$. The first experiments in [1], [3] were executed over the same area but with $300 \mathrm{MHz}$ for a larger range spectrum overlap. 


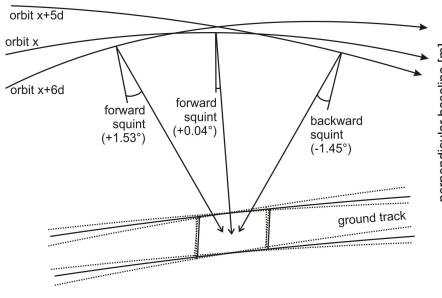

(a)

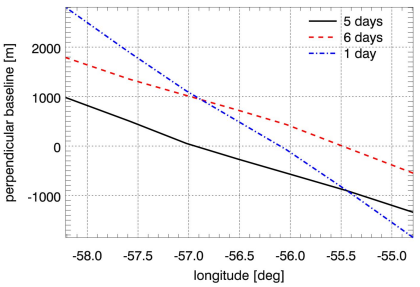

(b)
Fig. 1. (a) Acquisition geometry with crossing angles of $2^{\circ}$ and $4^{\circ}$ (b) Baselines of 3 interferograms with a 1-, 5- and 6-day lag over Ronne ice-shelf, Antarctica

Table 2. Time Series Acquisition Dates

\begin{tabular}{cccc}
\hline No. & 1. acq. date & 2. acq. date & 3. acq. date \\
\hline 0 & $2011-06-25$ & $2011-06-30$ & $2011-07-01$ \\
1 & $2011-08-19$ & $2011-08-24$ & $2011-08-25$ \\
2 & $2011-08-30$ & $2011-09-04$ & $2011-09-05$ \\
3 & $2011-09-10$ & $2011-09-15$ & $2011-09-16$ \\
4 & $2011-10-13$ & $2011-10-18$ & $2011-10-19$ \\
5 & $2011-10-24$ & $2011-10-29$ & $2011-10-30$ \\
6 & $2011-11-26$ & $2011-12-01$ & $2011-12-02$ \\
7 & $2011-12-29$ & $2012-01-03$ & $2012-01-04$ \\
8 & $2012-01-31$ & $2012-02-05$ & $2012-02-06$ \\
9 & $2012-03-04$ & $2012-03-09$ & $2012-03-10$ \\
10 & $2012-04-06$ & $2012-04-11$ & $2012-04-12$ \\
11 & $2012-05-09$ & $2012-05-14$ & $2012-05-15$ \\
\hline
\end{tabular}

\section{INTERFEROMETRIC RESULTS}

\subsection{Coherence and Coregistration}

The coherence of the 1-, 5- and 6-day time-lag interferograms from the acquisition period $13^{\text {th }}$ to $18^{\text {th }}$ Oct. 2011 is shown in Fig. 2 . The coherence dependency on the baseline is clearly visible. Approaching the zero baseline it is relatively high, before and after it becomes lower. This azimuth coherence trend of the consecutive interferograms matches perfectly with the baseline characteristics depicted in Fig. 1b, i.e., the zero baseline positions coincide. The fast loss of coherence follows from the quickly diverging orbit tracks, i.e. the increasing incidence angle difference results in an increasing volume decorrelation. A quantitative measurement of the coherence is given in Fig. 3, which shows the same trend for almost all 1- and 5-day interferograms. Geometric decorrelation was eliminated by azimuth adaptive spectral range filtering. This coherence behaviour is finally a limiting factor w.r.t. information extraction.

A key step in order to obtain the final interferograms and the true coherence is the coregistration. In a first step a geometric coregistration using an external DEM (in this case the ellipsoid) and the orbit is performed, which is usually sufficient for stationary scenes due the very precise orbit knowledge. Since the scene contains an unknown surface motion, an additional step is necessary, which consists in a conventional block-wise cross-correlation. Then the estimated block-wise offsets in azimuth and range are fitted with a weighted least squares algorithm into a second order polynomial, which is the information that will be used to further coregister the SLCs before the interferogram generation.

\subsection{Phase}

The fringe patterns in Fig. 2 show the phases between the 1-, 5- and 6-day lag acquisitions with the flat-earth phase removed. Despite the mostly low coherence in azimuth, fringes are perfectly visible in all interferograms. The fringes originate from geophysical features and are consistent with known surface velocity maps [6]. This was also verified by 11-day repeat-pass interferograms containing the same fringes [1].

The fringes in range arise from a gradient in the surface velocity of the glacier. Due to the lack of ground control points only a relative ground range velocity with a gradient of $0.2 \mathrm{~m} /$ day over the whole scene was obtained (cf. Section 4.2). The absolute velocity of around $2 \mathrm{~m}$ /day was already shown in [1], which was estimated by using the coregistration offsets. This value is in the same order of magnitude as the measurements presented in [6] and [7].

The fringes in azimuth can result from a reference height error, as no DEM was available. Only the WGS84 ellipsoid was used for the flat-earth removal. Up to now a mean ellipsoid height of $39.93 \mathrm{~m}$ was used, which results from averaging several height points of an ICESat data set. But a residual height offset together with the strongly varying baseline can still result in a linear phase ramp in azimuth, which should be equal for the 5- and 6-day interferogram but different for the 1-day one.

\section{CROSSING ORBITS TIME SERIES}

In this section the results of the time series are presented. The series consists of acquisition triplets and therefore three interferograms at each date (cf. Table 2).

\subsection{Coherence}

Fig. 3 shows the averaged coherence over range along azimuth (cf. with coherence figures in Fig. 2). In Fig. 3a the 1-day and in Fig. 3b the 5-day coherence is shown. The peak determines clearly the zero baseline position in azimuth. The larger volume decorrelation effect of the 1-day geometry causes the steeper slopes of the curves in Fig. 3a. But the 1-day coherence is principally more stable due to less temporal decorrelation. An outlier is the second 1-day acquisition pair on the $24^{\text {th }} / 25^{\text {th }}$ of August, 2011. The 5-day coherence suffers more from temporal decorrelation which causes variations in the coherence maximum as well as in the distribution along azimuth. The 6-day coherences show a similar distribution like the 5-day ones, except for a different zero baseline position in azimuth.

The maximum coherence of each interferogram is illustrated in Fig. 4. No clear seasonal trend is visible. The outliers are presumably due to temporal decorrelation resulting e.g. from weather influences like wind and snow. But an obvious trend is the lower 1-day coherence compared to the one of 5 and 6 days.

The typical error contributions to the coherence are

$$
\gamma \approx \gamma_{g e o m} \cdot \gamma_{k_{x}} \cdot \gamma_{v o l} \cdot \gamma_{t e m p} \cdot \gamma_{t h e r m} \cdot \gamma_{\text {proc }} \cdot \gamma_{a m b}
$$

where $\gamma_{\text {geom }}$ describes the geometric decorrelation, $\gamma_{k_{x}}$ the relative shift of the Doppler spectra, $\gamma_{v o l}$ the volume, $\gamma_{t e m p}$ the temporal, $\gamma_{\text {therm }}$ the thermal, $\gamma_{\text {proc }}$ the processing decorrelation and $\gamma_{a m b}$ decorrelation due to ambiguities. The geometric contributions in range and azimuth are principally accounted for in processing by azimuth varying common band filtering and instrument commanding, i.e. by applying squint angles to compensate the relative Doppler spectra shift. The volume decorrelation is supposed to be negligible due to looking at the zero baseline position. 

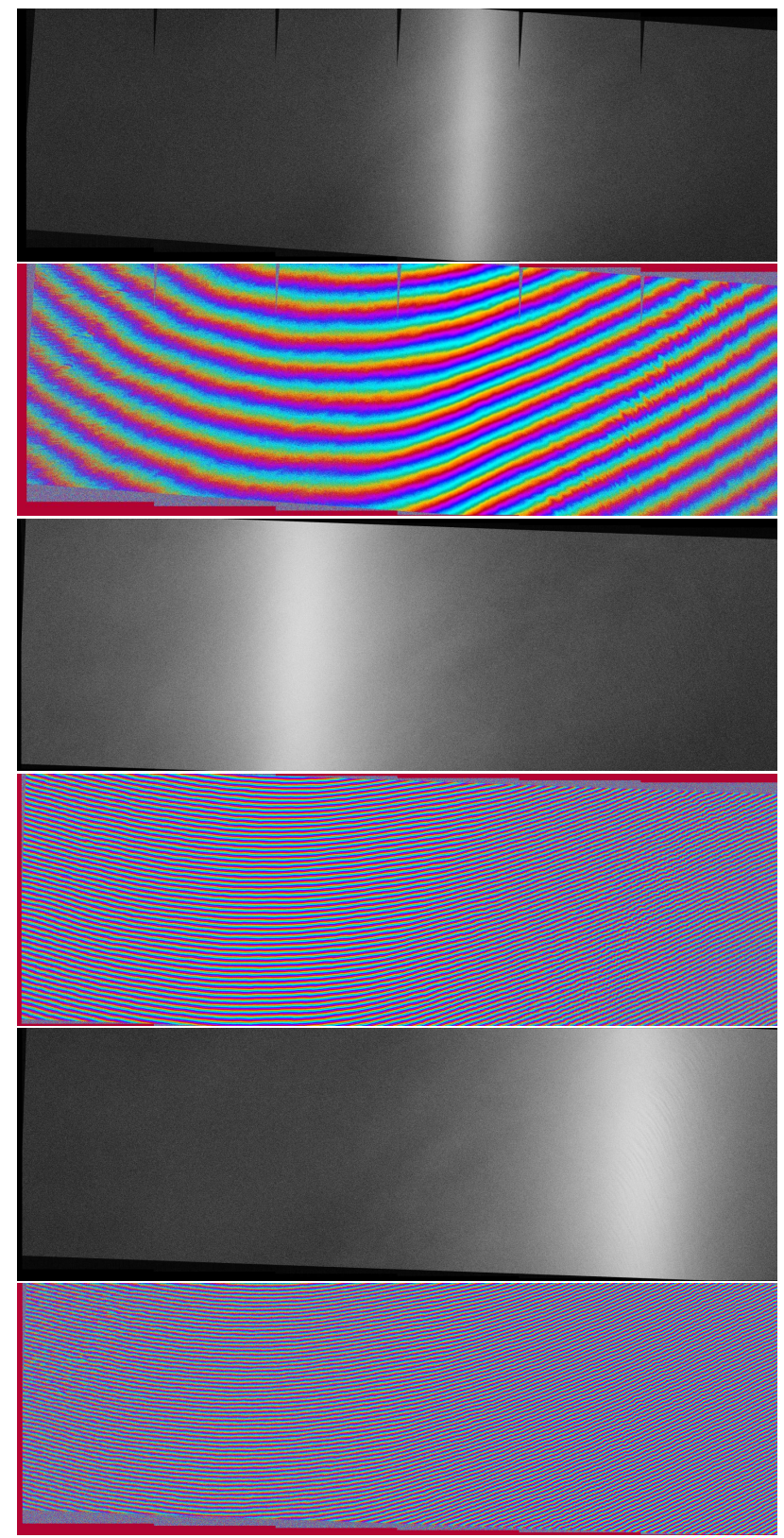

Fig. 2. Phase and coherence of crossing orbit interferograms over the Ronne ice-shelf acquired by the TDX satellite within the TSX mission. From top to bottom: 1-, 5-, 6-day lag interferogram, each with phase and coherence. Acquisition period $13^{\text {th }}-18^{\text {th }}$ Oct. 2011. The coherence range is from 0 to 0.8 (cf. Fig. 3). Dimensions: $80 \mathrm{~km}$ in azimuth, $30 \mathrm{~km}$ in range. (Range from bottom to top, Azimuth from right to left)

A supposedly large contribution is $\gamma_{a m b}$ due to the strongly squinted acquisitions. The relative squint of the 1-day interferogram is $2.98^{\circ}$ where for the 5- and 6-day interferogram it is only $1.49^{\circ}$ and $1.57^{\circ}$, respectively. It can be written as [8]

$$
\gamma_{a m b}=\frac{1}{\sqrt{\left(1+\mathrm{AASR}_{1}\right)\left(1+\mathrm{AASR}_{2}\right)}},
$$

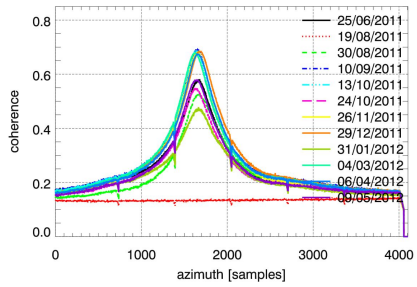

(a)

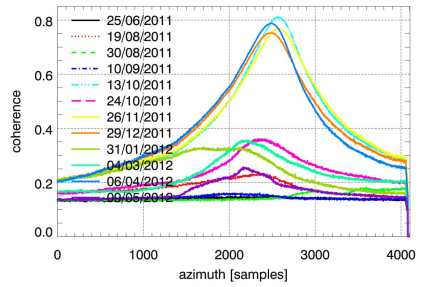

(b)
Fig. 3. Range-averaged coherence for all interferograms of the time series. (a) Coherence of 1-day lag and (b) 5-day lag interferograms.

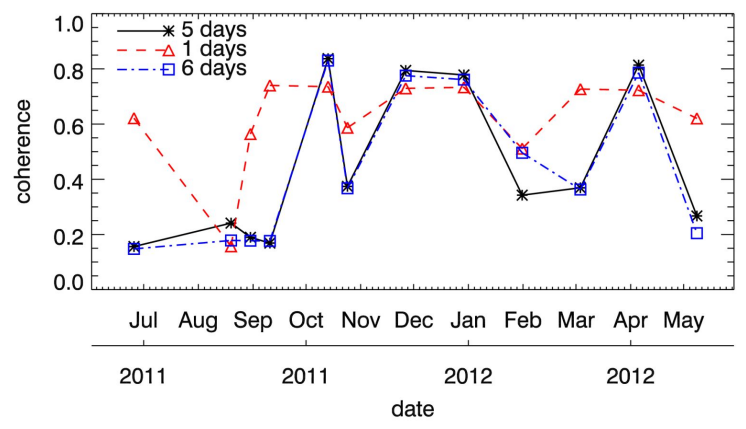

Fig. 4. Maximum coherence of each interferogram of the time series. Data sets corrected w.r.t. AASR.

where $\mathrm{AASR}_{\mathrm{n}}$ denotes the azimuth ambiguity to signal ratio of the first and second acquisition, respectively. Using (2), the 1-, 5- and 6-day coherence in Fig. 4 was already corrected by the factors 0.936 , 0.97 and 0.961 respectively.

Despite this consistent correction, the explanation of the remaining deviation between 1 and 5/6 days is still under investigation. The azimuth varying common band filtering as well as the coregistration accuracy, contributing to $\gamma_{p r o c}$, might be possible factors to be improved. The temporal decorrelation is supposed to be more eventdriven, like e.g. snow storms, which can explain outliers, but not a regular offset between the 1- and 5/6-day coherence. The probability w.r.t. $\gamma_{t e m p}$ of 5 and 6 days being lower than in the 1 day is obviously higher. This can be also observed in all drops in Fig. 4.

\subsection{Surface Velocity}

The particular test site on the Ronne ice-shelf was chosen due to its flat topography. The Ronne ice-shelf is well known for its glacier surface motion [6]. This leads to the assumption that the regular fringe patterns, visible in Fig. 2 and Fig. 5, show a changing velocity over the glacier surface mainly along the range direction. Note, that the fringe frequency is always similar, as shown in the three examples in Fig. 5. In Fig. 6 this relative velocity of the whole scene of around $0.2 \mathrm{~m} /$ day is shown. It results from the total unwrapped phase of the 5-day lag phase of Fig. 2 normalized to 1 day.

Fig. 7 shows this velocity difference across the scene for all acquisitions of the time series. The difference is normalized to one kilometer in ground range as the different acquisition pairs have slightly different range sizes. Considering only the azimuth position of highest coherence, no residual topography is supposed to have an 


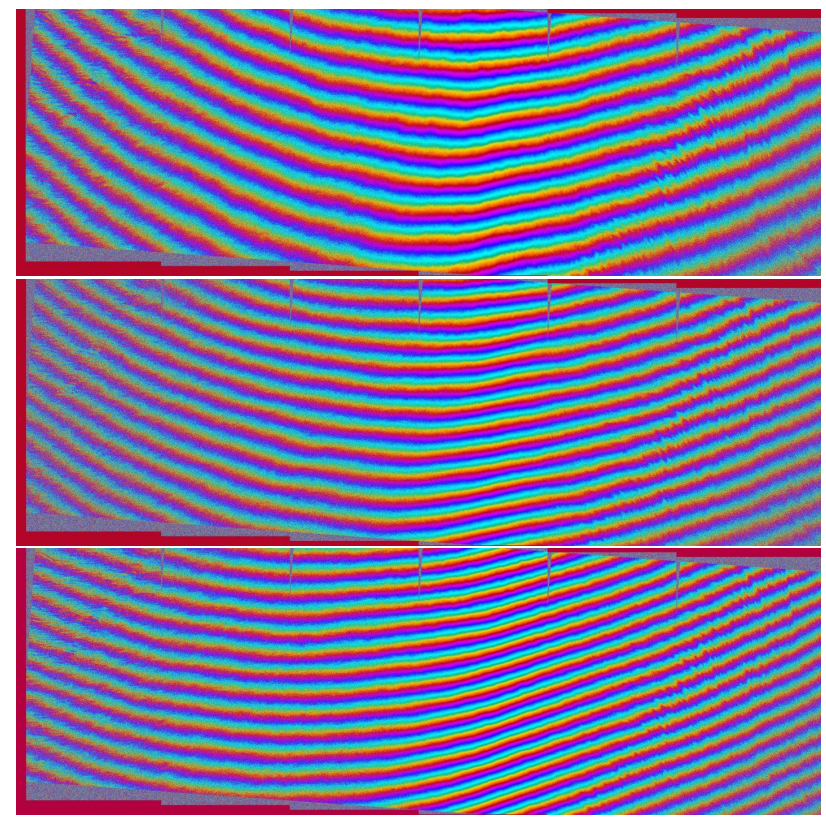

Fig. 5. Phases of 1-day lag interferograms. From top to bottom: Acquisitions no. 7, 8, 9 (cf. Table 2). (Range from bottom to top, Azimuth from right to left)

impact on the velocity measurement at all. The measurements of the three different day lags match quite good and show an average velocity difference of $1.25 \mathrm{~cm} / \mathrm{day} / \mathrm{km}$. The velocity seems to show a smooth trend in the second half of the acquisition period, which might be due to tidal motion and the regular date sampling. The regular offset between the 5- and 6-days measurements probably results from the different azimuth positions of the zero baseline together with the slightly different fringe pattern at this positon at which the velocity difference is calculated. Note, that the second measurement of the 1-day lag could not be calculated due to a weak fringe pattern.

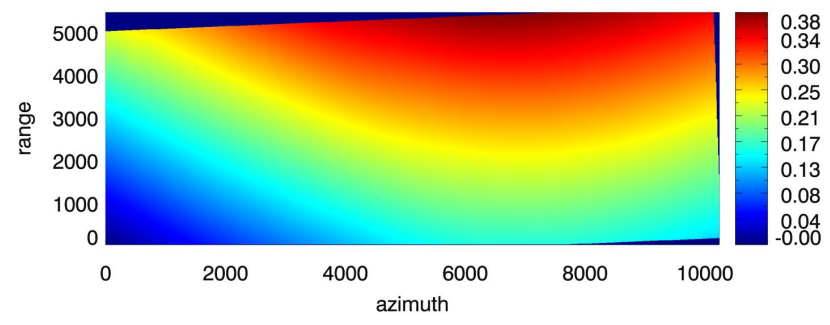

Fig. 6. Relative surface velocity of test site. Derived from total unwrapped 5-day phase. Normalized to 1 day, i.e. in [m/day].

\section{CONCLUSION}

The crossing orbit acquisition technique allows for 1-, 5- and 6-day quasi repeat-pass interferograms with TSX. The desired interferograms show promising results w.r.t. coherence investigations and surface motion measurements. A coherence evaluation of the one year time series of these short-term interferograms over the Ronne ice-shelf could not show any seasonal trend. However, it shows a

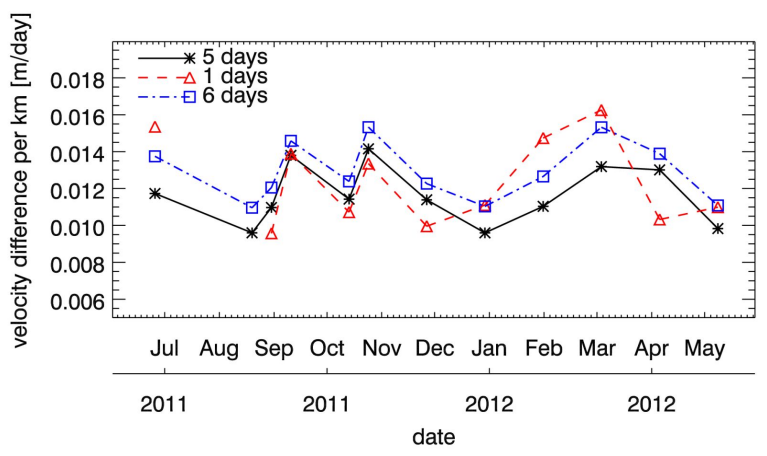

Fig. 7. Velocity difference per $\mathrm{km}$ across ground range of each interferogram of the time series.

more event-driven behavior, i.e. a dependency on changing weather conditions for instance. Further investigations on the coherence are planned including differences of the 1- and 5/6-day coherences and e.g. the volumetric coherence. Moreover, an evaluation of the phase resulted in a surface motion gradient across the scene which seems to have a very small, but smooth trend partly during the year. Additional investigations w.r.t. the fringe pattern, the reference height and tidal motion have to be conducted.

\section{REFERENCES}

[1] S. Wollstadt, P. López-Dekker, P. Prats, F. De Zan, T. Busche, and G. Krieger, "Interferometric Crossing Orbit Experiment using TerraSAR-X and TanDEM-X," in Proc. of Fringe 2011 Workshop, Frascati, Italy, 2011.

[2] P. López-Dekker, P. Prats, F. De Zan, S. Wollstadt, D. Schulze, G. Krieger, and A. Moreira, "Demonstration of SAR interferometry under crossing orbits using TerraSAR-X and TanDEM$\mathrm{X}$," in Proc. IEEE International Geoscience and Remote Sensing Symposium (IGARSS), Vancouver, Canada, Jul. 2011.

[3] P. López-Dekker, P. Prats, F. De Zan, D. Schulze, G. Krieger, and A. Moreira, "TanDEM-X first DEM acquisition: a crossing orbit experiment," IEEE Geosci. Remote Sens. Lett., vol. 8, no. 5, pp. 943-947, May 2011.

[4] F. Gatelli, A. Monti Guarnieri, F. Parizzi, P. Pasquali, C. Prati, and F. Rocca, "The Wavenumber Shift in SAR Interferometry," IEEE Trans. Geosci. Remote Sens., vol. 32, no. 4, pp. 855-865, Jul. 1994.

[5] A. K. Gabriel and R. M. Goldstein, "Crossed orbit interferometry: theory and experimental results from SIR-B," International Journal of Remote Sensing, vol. 9, no. 5, pp. 857-872, 1988.

[6] E. Rignot, J. Mouginot, and B. Scheuchl, "Ice Flow of the Antarctic Ice Sheet," Science doi 10.1126/science.1208336, 2011.

[7] D. G. Vaughan and M. Jonas, "Measurements of velocity of Filchner-Ronne Ice Shelf," AWI, Bremerhaven, Filchner-Ronne Ice Shelf Programme (FRISP) Report No. 10, 1996.

[8] G. Krieger, A. Moreira, H. Fiedler, I. Hajnsek, M. Werner, M. Younis, and M. Zink, "TanDEM-X: A Satellite Formation for High-Resolution SAR Interferometry," IEEE Trans. Geosci. Remote Sens., vol. 45, no. 11, pp. 3317-3340, Nov. 2007. 